\title{
Innovations in Non-Linear Oscillations of a Pendent Drop From a Capillary Tip during Formation and Detachment - An LBM Simulation
}

\author{
S. Ghorbanifar ${ }^{1}$, M. Taeibi Rahni ${ }^{2,3 \dagger}$ and M. Zareh ${ }^{1}$ \\ ${ }^{1}$ Islamic Azad University Science and Research Branch, Tehran, Iran \\ ${ }^{2}$ Aerospace Department, Sharif University of Technology, Tehran, Iran \\ ${ }^{3}$ ARI, Ministry of Higher Education, Tehran, Iran \\ $\dagger$ Corresponding Author Email: taeibi@sharif.eduAbstract
}

(Received January 4, 2020; accepted July 9, 2020)

\begin{abstract}
Individual drops are suitable tools to study the liquid-fluid interfacial properties. In this work, forcedisplacement equation and non-linear oscillations of a pendent drop are numerically investigated. The presented novel force-displacement function allows following the dynamics of a pendent drop and realizing its elastic behavior. The growth and detachment of drop, which is pending due to gravity from a capillary tip, is considered (assuming high density and high viscosity ratios and immiscible two-phase flows). Twodimensional multi-relaxation time lattice Boltzmann method (MRT-LBM) was used to simulate growth, detachment, and oscillations of the drop using a conservative model for high-density ratio. The forcedisplacement function of a pendent drop (FDFPD), which is non-linear, was introduced. Using FDFPD, the non-linear elastic specifications of the pendent drop were determined. It was realized that the drop shows three different elastic behaviors simultaneously (hardening, linear, and softening). The drop superharmonic and subharmonic frequencies were calculated, using the natural frequency of the linear portion of FDFPD. Besides, the drop would grow as long as its displacement is between the extrema of FDFPD. In addition, a dynamic criterion for the onset of detachment was established. Also, increasing the Bond number from 0.11 to 1.96 , while keeping Reynolds number equal to 0.023 , accelerates the drop detachment and increases the linear portion of FDFPD. It was shown that increasing Capillary number from $1.8 \mathrm{E}-5$ to $7.3 \mathrm{E}-4$, while keeping Reynolds number equal to 0.023 , accelerates the drop detachment and increases the non-linear portions of FDFPD.
\end{abstract}

Keywords: Two-phase flow; Growing drop; Drop non-linear oscillations; Lattice Boltzmann method; Drop dynamics

\section{NOMENCLATURE}

$\begin{array}{lll}\text { Bo } & \text { Bond number } & \\ \text { Ca } & \text { Capillary number } & \mathrm{N}_{\mathbf{y}} \\ c_{s} & \text { speed of sound in the system } & \hat{\mathbf{n}} \\ \mathrm{D} & \text { inner pipe diameter } & \hat{\mathbf{n}}_{\mathrm{w}} \\ \mathbf{e}_{\alpha} & \text { macroscopic velocity set } & p \\ F_{\alpha} & \text { forcing term } & p_{i n} \\ \mathbf{F} & \text { external force vector exerted to the fluid } & p_{i n} \\ \mathbf{F}_{b} & \text { body force vector } & R \\ \mathbf{F}_{s} & \text { surface tension force vector } & \mathrm{Re} \\ f(y) & \text { force-displacement function of a pendent } & \hat{\mathbf{S}} \\ & \text { drop (FDFPD) } & t \\ \mathbf{G} & \text { gravitational acceleration vector } & t^{*} \\ \bar{g}_{\alpha} & \text { hydrodynamic distribution function } & \mathbf{u} \\ h_{\alpha} & \text { phase-field distribution function } & W \\ \bar{k} & \text { spring constant of the linear portion of the } & w_{\alpha} \\ & \text { FDFPD } & \mathbf{x} \\ \bar{m} & \text { average drop mass } & \mathbf{x}_{0} \\ \mathrm{M} & \text { system mobility } & \\ \mathbf{M} & \text { orthogonal transformation } & X_{c} / D \\ \mathrm{~N}_{\mathrm{x}} & \text { domain resolution, the horizontal } & y\end{array}$

direction

domain resolution, the vertical direction interface unit normal vector wall unit normal vector macroscopic pressure inside pressure of drop outside pressure of drop radius of the drop Reynolds number diagonal relaxation matrix time dimensionless time macroscopic velocity vector interface thickness weight coefficients position vector initial position vector of the equilibrium phase-field profile dimensionless drop mass center independent variable of FDFPD 
$\alpha \quad$ direction index

$\varphi \quad$ diffuse-interface phase-field variable

$\varphi_{\mathrm{w}} \quad$ wall phase-field variable

$\Omega_{\alpha} \quad$ collision operator

$\mu \quad$ local fluid viscosity

$\mu_{\varphi} \quad$ chemical potential for the binary fluids

$v \quad$ kinematic viscosity

$\rho \quad$ local fluid density

\section{$\rho_{H} \quad$ heavier phase density}

$\rho_{L} \quad$ lighter phase density

$\tau \quad$ hydrodynamic relaxation time

$\tau_{\varphi} \quad$ phase-field relaxation time

$\tau_{H} \quad$ heavier phase relaxation time

$\tau_{L} \quad$ lighter phase relaxation time

$\theta \quad$ contact angle at the solid boundary

$\sigma \quad$ surface tension

\section{INTRODUCTION}

Liquid-fluid interactions are omnipresent in the real-world, namely nature and industry. Therefore, individual drops are often used for the specification of liquid-fluid interfacial properties (Miller and Liggieri 2011). Their study helps analyze systems with similar interfaces or the same dynamics. As an example of the latter case, one can refer to a dripping faucet, which is among the systems with non-linear oscillations and chaotic modality (d'Innocenzo and Renna 1996; Shaw 1984). Also, oscillating pendent drops constitute a suitable system for analyzing non-linear dynamics (DePaoli et al. 1995). Non-linear elastic behavior of oscillatory drops has convinced many researchers that the study of the vibrating drop is beneficial, in order to describe systems with non-linearity and chaotic nature (Shaw 1984). Many experimental, analytical, and numerical approaches exist that have tried to tackle such a problem. Among them, numerical approaches have been increasingly used. Most works done in this field have been accompanied by the omission of dynamic effects and assuming thermodynamic equilibrium. Nevertheless, those efforts have resulted in important and applicable outcomes. More recently, the main interest of researchers in this area has focused on the dominant interactions of dynamical effects. Besides, phases in liquid-fluid systems have complex dynamic characters, which demand theoretical and practical treatments (Sultana et al. 2017). That is why investigations of drop formation, oscillation, and detachment from a capillary tip have special importance.

\subsection{Conventional CFD Simulations of Drop Dynamics}

Let us consider drop dynamics as a challenging problem in hydrodynamics, which has affected the complexity of the mutual effects of liquid-fluid interfaces. In 1997, drop formation at the tip of a vertical tube, immersed in a second immiscible fluid, was numerically studied (Zhang and Stone 1997) at low Reynolds number of the flow, using boundary integral method. Zhang and Stone examined the influence of some dimensionless numbers on evolution, breakup of drops, and the size of separated satellites. They found that for viscosity ratios smaller than 10 , no large satellite drops are formed. They also imposed external uniform flow to the system, which reduced the size of the drop. One of their significant results belongs to the effect of nondimensional Capillary and Bond numbers on drop size and its break up. In 1998, a novel axisymmetric dynamical model of drop detachment in gas metal arc welding was presented (Jones et al. 1998). Jones et al. compared the numerical results with the experimental measurements of welding images. They reached valuable results about the variable surface tension of drops. They made a qualitative observation about the bouncing force exerted by the detached drop to the upstream of the flow. Also, this phenomenon was reported previously in 1994 (Miller et al. 1998) during drop detachment measurements. In 2000, (Lau and Mashayek 2001) investigated oscillating drop warming using numerical simulation of hot gas environments. Lau and Mashayek observed that the most apparent feature of thermo-capillary flows was demonstrated by vortices. The number and strength of vortices were varied with the mode of temperature disturbance. In 2003, (Davidson and Copper-White 2003) used VOF numerical simulation to predict the dynamics of shear-thinning liquid drop formation in the air from a circular orifice. They examined the effect of Weber, Froude, and Ohnesorge Numbers on drop shape, pinch-off, and thickness length. Drop formation at a capillary tip and in a channel flow was investigated by (Cramer 2004). Cramer realized that there are mainly five important dimensionless numbers engaged in the physics of this problem, namely: Capillary, Ohnesorge, Reynolds, Bond, and viscosity ratio. Moreover, he studied periodic dripping, which was previously studied by (Fainerman and Miller 1995). Cramer concluded that after drop pinch-off, the residual fluid upstream starts to vibrate and transfer vibration to the newly forming drop, which in turn affects its detachment. This result had been confirmed by (Jones et al. 1998). In 2005, Fawehinmi et al. (Fawehinmi et al. 2005), who performed a numerical simulation using commercial CFD packages by implementing the VOF method to study drop formation. Also, he made an experimental study comparing the two results. They argued that experimental results revealed for the same grid resolution, the single and multiphase solvers offer similar accuracy of prediction and are both able to capture the features of most significant practical engineering interest, such as primary drop volume as a function of flow rate. Using first-order time discretization, the formation of oil drops both numerically and experimentally were studied from a single capillary tube with cross-flow, using continuous water phase by (Timgren et al. 2009). They concluded that increasing cross-flow velocity decreases the size of the drop and vice versa. The linear problem of axisymmetric, inviscid, volume-preserving 
vibrations of a liquid drop, pinned along a latitude was studied by (Bostwick and Steen 2009). They solved this problem using numerically generated eigenfrequencies for their integrodifferential boundary value problem. Considering the center of mass, they used the effect of a pinned drop to introduce a new low-frequency eigenmode. However, during their precise work, they only considered linear oscillations. In 2013, drop formation and pinch-off from a capillary tube under the influence of gravity was widely studied by (Bierbrauer et al. 2013), using FEM. They studied the critical pinch-off time required for drop ejection. They found that the required ejection time decreases exponentially with average pipe velocity. However, the lack of drop oscillations was highlighted in their study. An extensive experimental and numerical study on drop formation, oscillation, and detachment was performed by (Karbaschi et al. 2015). Their study was mainly experimental measurements of drop surface tension coefficient. However, they did not report any results related to the natural frequency of drops. One of the recent works has been performed by (Nazari et al. 2018). In this experimental work, they investigated the growing drop profiles using a high-speed camera and generated a curve that relates the drop mass center to the time during drop growth and formation. Nowadays, researchers' attention has been switched towards modern meshless CFD methods (e.g., LBM). Due to the intrinsic mechanism of LBM, which has its basis in mesoscale, it has increasingly gained success in multiphase flows. One reason may be the fact that the hydrodynamic interactions of multiphase interfaces are governed by molecular scales and are best fitted with LBM nature, rather than with conventional CFD methods (a macro-scale approach).

\subsection{LBM Simulation of Drop Dynamics}

In the past one and half decades, many works have been performed using LBM for single drop dynamics and its behavior. LBM was used by (Fakhari and Rahimian 2009), to simulate a drop subjected to a gas stream (using Reynolds and Weber numbers). One year later, they investigated the free fall of a drop in an axisymmetric pipe by studying surface tension effects in conjunction with impacts of gas and drop viscosity via, studying the effect of Eotvos, Morton, and Archimedes numbers (Fakhari and Rahimian 2010). Later in 2011, they extended their falling drop simulation to MRTLBM (Fakhari and Rahimian 2011). In the years 2013 to 2017, (Fakhari and Bolster 2017; Fakhari et al.2016; Fakhari and Lee 2013; Geier et al.2015) developed LBM model for multiphase flows, namely: conservative phase-field model.

Despite the extensive studies on growing drops, a complete description of the drop elastic behavior (during the growth, formation, and detachment process) has not yet been performed. The novelty of this work is to numerically obtain an algebraic relation for a drop elastic deformation during growth and detachment, using LBM. For the pendent drop, the proposed equation (FDFPD) unifies different drop behaviors (hardening, linear, and softening; a very rare phenomenon that occurs in the growth of a pendent drop) all in one continuous non-linear function. While, other methods have linearized the drop elastic behavior by splitting the drop into two or more parts to study the force-displacement relation. Also in this work the profiles of the growing drop, along with its mass, velocity, and pressure have been captured numerically which can be used to evaluate its natural, super-harmonic, and subharmonic frequencies. While, in other works fast cameras have been used to capture the instantaneous drop profile, without any information about its mass, velocity, and pressure. In addition, there have not been reported any numerical works concerning the frequency evaluation of the growing pendent drops.

\section{PHYSICAL MODEL}

In this work, we have presented two-dimensional simulation of formation, oscillation, and detachment of a single pendent drop at a capillary tip. The fluid is assumed isothermal, Newtonian, incompressible, and isotropic. The flow entering the domain from the pipe tip is uniform and steady (time has no impact on the non-linearity of the drop), with zero mass transfer between the two phases. In the beginning, there is no dense phase in the region, and the drop growth starts with the injection of liquid. Continuing this process eventually leads to drop detachment. Figure 1 shows schematic representation of this physical model.

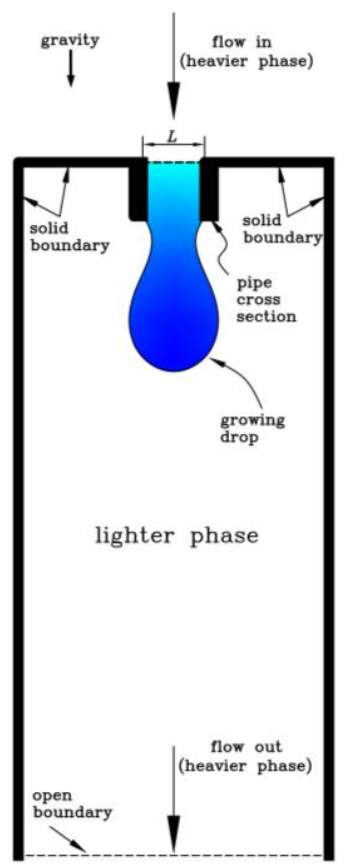

Fig. 1. Schematic of the flow geometry with a pendent drop.

\section{NUMERICAL MODEL}

Fakhari and his coworkers have developed an LBM model for multiphase flows, named conservative 
phase-field model. In this section, a brief explanation of interface capturing and the hydrodynamic equations used in the conservative phase-field model are presented.(Fakhari and Bolster 2017; Fakhari et al. 2016; Fakhari and Lee 2013; Geier et al. 2015).

\subsection{Interface Capturing Equation}

There exist two well-known approaches for interface capturing, namely the CahnHilliard equation (Cahn and Hilliard 1958) and Allen-Cahn equation (Allen and Cahn 1979). For Cahn-Hilliard equation, regardless of its mass conserving property, one should discretize a fourthorder derivative term, which demands careful attention. While, Allen-Cahn equation only includes second-order derivative terms. Interestingly enough, Chiu and Lin (Chiu and Lin 2011) combined both advantages of Cahn-Hilliard, and Allen-Cahn equations.

In the conservative model introduced by (Geier et al. 2015) for a system containing incompressible binary-fluid, the governing equation for interface tracking is the following Allen-Cahn equation:

$$
\begin{aligned}
& \partial \varphi / \partial t+\nabla \cdot(\varphi \mathbf{u}) \\
= & \nabla \cdot[M(\nabla \varphi-(4 / W) \varphi(1-\varphi) \hat{\mathbf{n}}),
\end{aligned}
$$

where $\varphi$ is the diffuse-interface phase-field variable with zero value in the lighter phase and one in the heavier phase, $\mathbf{u}$ is macroscopic velocity vector, $t$ is time, $W$ is the interface thickness, $M$ is mobility, and $\hat{\mathbf{n}}$ is the interface unit normal vector, so that its positive direction is towards the heavier phase. Thus,

$$
\hat{\mathbf{n}}=\frac{\nabla \varphi}{|\nabla \varphi|} .
$$

Also, $\varphi) \mathbf{x}($ is assumed to change as follows:

$$
\varphi(\mathbf{x})=\frac{1}{2}\left\lfloor 1-\tanh \left(\frac{\left|\mathbf{x}-\mathbf{x}_{0}\right|}{W / 2}\right)\right\rfloor,
$$

in which equilibrium phase-field profile is located at $\mathbf{x}_{0}$ and is used as an initial condition for $\varphi$ derived by minimizing the system bulk energy (Lee 2009). Jacqmin (Jacqmin 2000) proposed a wetting condition, to impose a contact angle, $\theta$, at a solid boundary. Note $\varphi_{\mathrm{w}}$ is the wall phase-field and $\hat{\mathbf{n}}_{\mathrm{w}}$ is the wall unit normal vector, i.e.,

$\left.\hat{\mathbf{n}}_{w} \cdot \nabla \varphi\right|_{\mathbf{x} w}=\Theta \varphi_{w}\left(1-\varphi_{w}\right)$,

$\Theta=-\sqrt{\frac{2 \beta}{\kappa}} \cos \theta$,

$\kappa=3 \sigma \frac{W}{2}$,

$\beta=\frac{12 \sigma}{W}$,

and $\sigma$ is surface tension coefficient.

\subsection{Hydrodynamic Equations}

For an incompressible, isothermal, multiphase flow, Navier-Stokes equations are (Clift et al. 1978):

$\frac{\partial \rho}{\partial t}+\nabla \cdot \rho \mathbf{u}=0$

$\rho\left(\frac{\partial \mathbf{u}}{\partial t}+\mathbf{u} \cdot \nabla \mathbf{u}\right)=-\nabla p$

$+\nabla \cdot\left(\mu\left[\nabla \mathbf{u}+(\nabla \mathbf{u})^{T}\right]\right)+\mathbf{F}$,

where $\rho$ is the local fluid density, $\mu$ is the local fluid viscosity, and $p$ is the macroscopic pressure. Also, $\mathbf{F}$ is the external force exerted to the fluid. In this study, the force consists of two components as:

$\mathbf{F}=\mathbf{F}_{s}+\mathbf{F}_{b}$

where $\mathbf{F}_{s}$ and $\mathbf{F}_{b}$ are surface tension and body forces, respectedly. Jacqmin (Jacqmin 1999) proposed the following relation for surface tension force:

$\mathbf{F}_{s}=\mu_{\varphi} \nabla \varphi$,

where $\mu_{\varphi}$, the chemical potential for the binary fluids, is defined as:

$\mu_{\varphi}=4 \beta \varphi(\varphi-1)(\varphi-1 / 2)-\kappa \nabla^{2} \varphi$.

$\mathbf{F}_{b}$ is written as:

$\mathbf{F}_{b}=\left(\rho_{H}-\rho_{L}\right) \mathbf{G}$,

where $\mathbf{G}$ is the gravitational acceleration, while $\rho_{H}$ and $\rho_{L}$ are heavier and lighter phase densities, respectively. Note, the densities, and the forces are local.

\section{NUMERICAL METHOD}

Here, we used conservative phase-field LBM proposed by (Mitchell et al. 2018) to solve the interface tracking and hydrodynamics equations. It has been proven that the following LBE can recover Eq. (1) for conservative phase-field (Krüger et al. 2017), as:

$h_{\alpha}\left(\mathbf{x}+\mathbf{e}_{\alpha} \Delta t, t+\Delta t\right)=h_{\alpha}(\mathbf{x}, t)$

$-\frac{h_{\alpha}(\mathbf{x}, t)-h_{\alpha}^{e q}(\mathbf{x}, t)}{\tau_{\varphi}+1 / 2}$,

where $\tau_{\varphi}$ is phase-field relaxation time, $h_{\alpha}$ is phasefield distribution function, and $\mathbf{e}_{\alpha}$ is macroscopic velocity set.

In this model, the equilibrium distribution function for the phase-field is:

$h_{\alpha}^{e q}=\varphi \Gamma_{\alpha}$
$+w_{\alpha} \frac{M}{c_{s}^{2}}\left[\frac{4}{w} \varphi(1-\varphi)\right]\left(\mathbf{e}_{\alpha} \cdot \hat{\mathbf{n}}\right)$, 


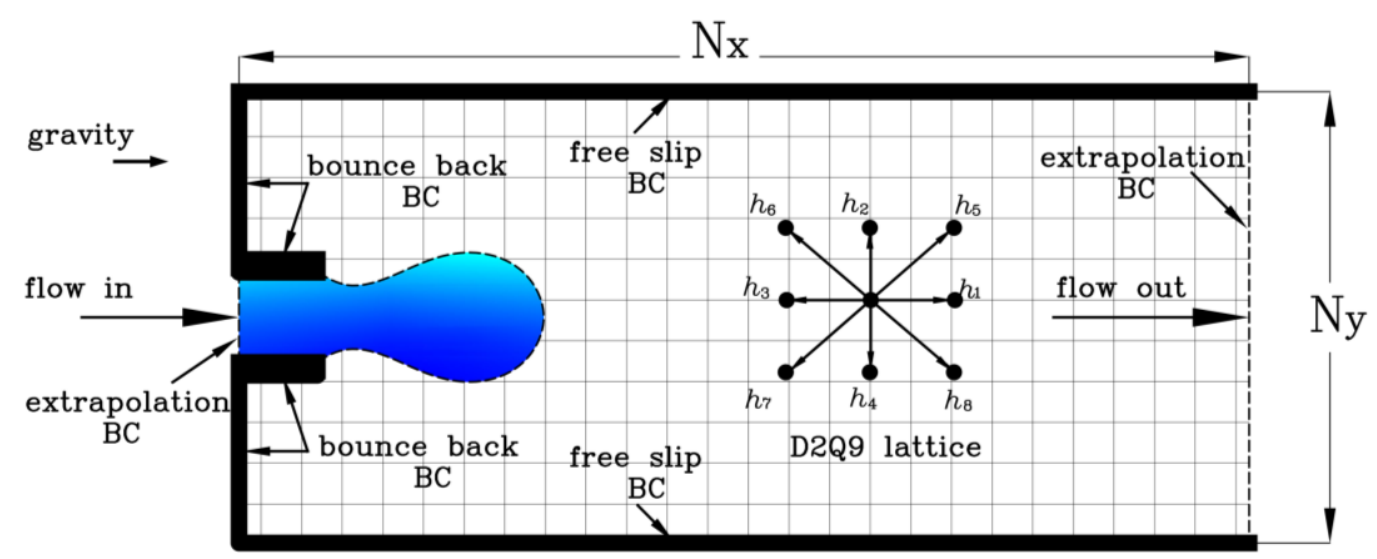

Fig. 2. Boundary conditions used in the computational domain.

where

$$
\Gamma_{\alpha}=w_{\alpha}\left\lfloor 1+\frac{\mathbf{e}_{\alpha} \cdot \mathbf{u}}{c_{s}^{2}}+\frac{\left(\mathbf{e}_{\alpha} \cdot \mathbf{u}\right)^{2}}{c_{s}^{4}}-\frac{\mathbf{u} \cdot \mathbf{u}}{2 c_{s}^{2}}\right\rfloor,
$$

and $c_{s}=c / \sqrt{3}$ is speed of sound, $w_{\alpha}$ is weight coefficients, and $\mathrm{M}$ is system mobility, which is related to the relaxation time as (Mitchell et al. 2018):

$$
M=\tau_{\varphi} c_{s}^{2} \Delta t
$$

For D2Q9 lattice, macroscopic velocity set and associated weight coefficients are listed in the Appendix. Eq. (11) is solved in two steps: collision and streaming. After streaming step, phase-field is updated as:

$\varphi=\sum_{\alpha} h_{\alpha}$.

Density is calculated using the following relation:

$\rho=\rho_{L}+\varphi\left(\rho_{H}-\rho_{L}\right)$.

For an almost incompressible multiphase flow, lattice Boltzmann equation is (Fakhari et al. 2017):

$$
\begin{aligned}
\bar{g}_{\alpha}\left(\mathbf{x}+\mathbf{e}_{\alpha} \Delta t\right. & , t+\Delta t)=\bar{g}_{\alpha}(\mathbf{x}, t) \\
& +\Omega_{\alpha}(\mathbf{x}, t)+F_{\alpha}(\mathbf{x}, t),
\end{aligned}
$$

where, $\bar{g}_{\alpha}$ is hydrodynamic distribution function and $\Omega_{\alpha}$ is a collision operator. Again, this equation can be solved using the collision-streaming approach. In Eq. (17), $F_{\alpha}$ is a forcing term given as (Fakhari and Bolster 2017):

$$
\begin{aligned}
F_{\alpha}=\Delta t[( & \left.\Gamma_{\alpha}-w_{\alpha}\right)\left(\rho_{H}-\rho_{L}\right) c_{s}^{2} \\
& \left.+\Gamma_{\alpha} \mu_{\varphi}\right]\left(\mathbf{e}_{\alpha}-\mathbf{u}\right) \cdot \Delta \varphi \\
& +\Delta t \Gamma_{\alpha}\left(\mathbf{e}_{\alpha}-\mathbf{u}\right) \cdot \mathbf{F}_{b} .
\end{aligned}
$$

Using the MRT model for collision operator, $\Omega_{\alpha}$ is:

$$
\Omega_{\alpha}=-\mathbf{M}^{-1} \hat{\mathbf{S}} \mathbf{M}\left(\bar{g}_{\alpha}-\bar{g}_{\alpha}^{e q}\right),
$$

Where,

$\bar{g}_{\alpha}^{e q}=g_{\alpha}^{e q}-\frac{F_{\alpha}}{2}$,

and

$g_{\alpha}^{e q}=p w_{\alpha}+\rho c_{s}^{2}\left(\Gamma_{\alpha}-w_{\alpha}\right)$,

while, $\mathbf{M}$ is an orthogonal transformation, which maps physical space onto momentum space, and $\hat{\mathbf{S}}$ is diagonal relaxation matrix and is listed in the Appendix for D2Q9 lattice.

After solving Eq. (17) and obtaining $\bar{g}_{\alpha}$, one can calculate hydrodynamic properties, using the following equations (Fakhari and Bolster 2017):

$$
\begin{aligned}
& \mathbf{u}=\frac{1}{\rho c_{s}^{2}} \sum_{\alpha} \bar{g}_{\alpha} \mathbf{e}_{\alpha}+\frac{\Delta t}{2 \rho}\left(\mathbf{F}_{s}+\mathbf{F}_{b}\right), \\
& p=\sum_{\alpha} \bar{g}_{\alpha}+\frac{\Delta t}{2}\left(\rho_{H}-\rho_{L}\right) c_{s}^{2} \mathbf{u} \cdot \Delta \varphi .
\end{aligned}
$$

\section{BOUNDARY TREATMENT}

Figure 2 shows the computational domain, whose dimensions are $\mathrm{Nx}=192, \mathrm{Ny}=128, \mathrm{D}=14$ (inner diameter of the pipe), and $\mathrm{H}=10$ (height of the pipe).In the computational domain, three different boundary conditions have been used.

For input and output boundaries, the interpolation method proposed by Chen et al. (1996) was used. The main idea of the proposed method is to use a grid layer next to the input and output. The nonequilibrium part of the unknown distribution functions in these additional layers is calculated by extrapolating (quadratic) the values of the distribution function in the flow domain. For the pipe and left solid walls, a half-way bounce-back boundary condition was used. For the upper and lower walls, the free slip boundary condition was used. 


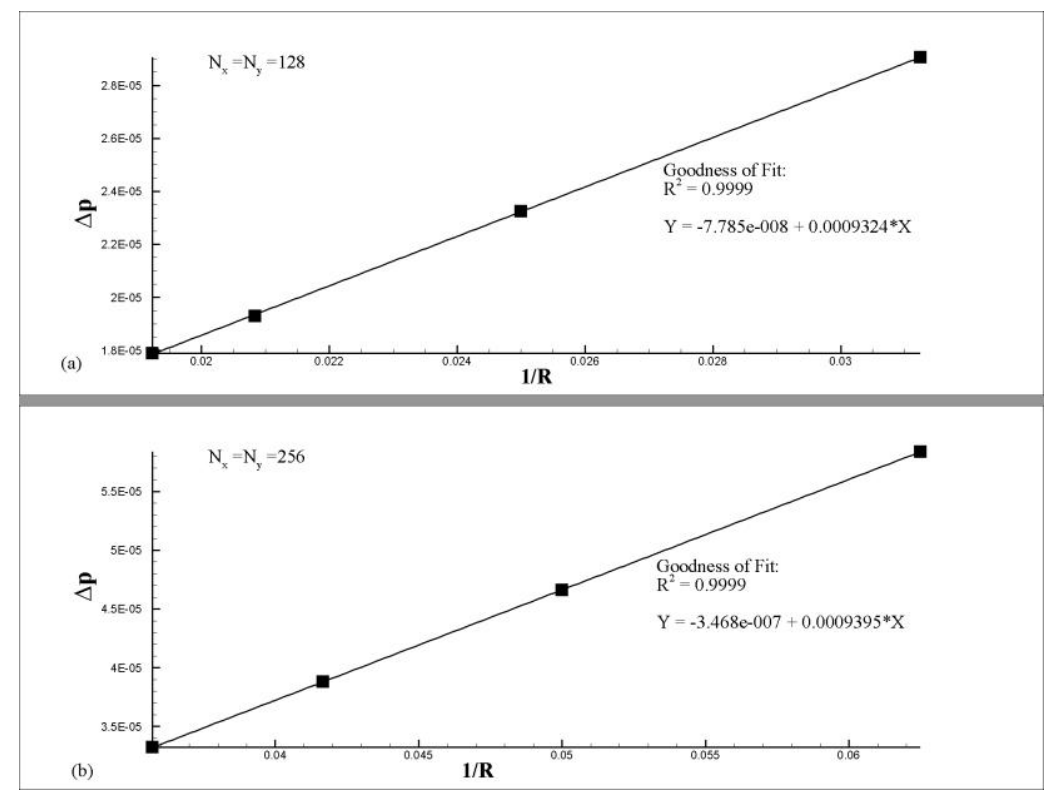

Fig. 3. Laplace test, (a) grid resolution of $128 * 128$, (b) grid resolution of $256 * 256$.

\section{VALIDATION}

\subsection{Numerical Method Validation}

The first criterion used is the Laplace test. This test was performed to study the code written for Boltzmann method. In this test, the numerical value of surface tension used in the code was calculated. According to Laplace's law, pressure difference inside and outside a two-dimensional drop follows the following relation:

$$
\Delta p=p_{\text {in }}-p_{\text {out }}=\frac{\sigma}{R}
$$

where, $\sigma$ is the surface tension, and $R$ is the radius of the drop. To examine the Laplace's law (all quantities are in lattice units), circular drops having radii equal to $16,20,24$, and 28 with $\sigma=0.001$ are located at the center of the computational domain (128*128 grids) with periodic boundary conditions. Also, by increasing the grid resolution, circular drops with radii of $32,40,48$, and 56 having $\sigma=0.001$ are located at the center of the computational domain $(256 * 256$ grids $)$ with periodic boundary conditions. After about 10,000 repetitions, the drop became utterly rounded. Note, the pressure values inside and outside the drop are calculated from Eq. (23). The results obtained from Eq. (24) are illustrated in Fig. 3 (mobility was set to 0.2 ).

The slope shown is the surface tension that is approximately equal to $0.000,9$. As can be seen, the numerical results are in agreement with the theoretical predictions, since all e points shown in the figure overlap the lines passing through the origin.

The second test was the square two-dimensional drop relaxation. For this purpose, a square drop with 80 grid units was positioned in the middle of a $240 * 240$ computational field. The periodic boundary conditions were used in the surrounding boundaries. Also, by increasing the grid resolution, a square drop with 160 grid units was positioned in the middle of a $480 * 480$ computational field.

The periodic boundary conditions were used in the surrounding boundaries.

The drop deformation changes with related domain density along a horizontal line passing through the middle of the drop. This is illustrated in Figs. 4 and 5 at some typical time steps.. The mobility was set to 0.2 .

\subsection{Results Validation}

Unlike linear springs, non-linear springs have variable spring coefficients (having variable elasticity). When stretching a spring, if the spring constant grows, it is called a hardening spring, and if it decreases, it is a softening spring. In Fig. 6, three regions of FDFPD with different characters are recognized. The first region (begins from almost the minimum of the FDFPD) belongs to the initial growth of the drop, which has hardening elasticity. Therefore, by increasing the force (due to increasing the weight of the drop), the surface tension force tends to increase such that by keeping its overall trend prevents the drop's necking. Of course, this resistance is not permanent, and there is an inflection point in FDFPD (beginning of the region with softening elasticity). From the inflection point to the maximum point of FDFPD, drop shows a softening elasticity. The drop detachment occurs at the maximum point of FDFPD. The region between hardening and softening regions is almost linear or pseudo-linear. As shown in Fig. 6, these three distinct behaviors of surface tension forces are due to geometrical changes in drop shape during the 


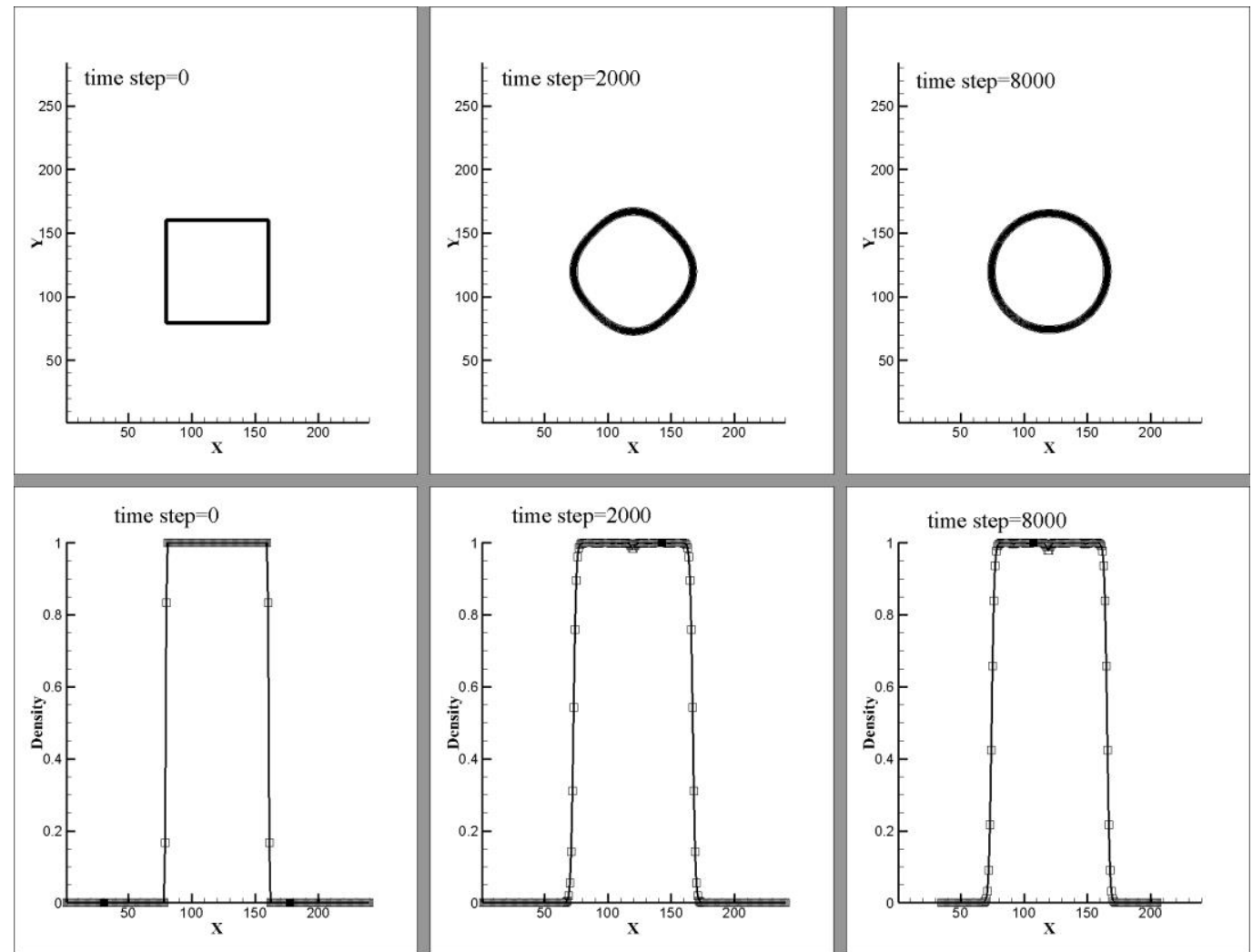

Fig. 4. Square drop relaxation test, drop shape evolution, and its density variation at the time steps $t=0$, 2000 , and 8000 for $240 * 240$ grid.

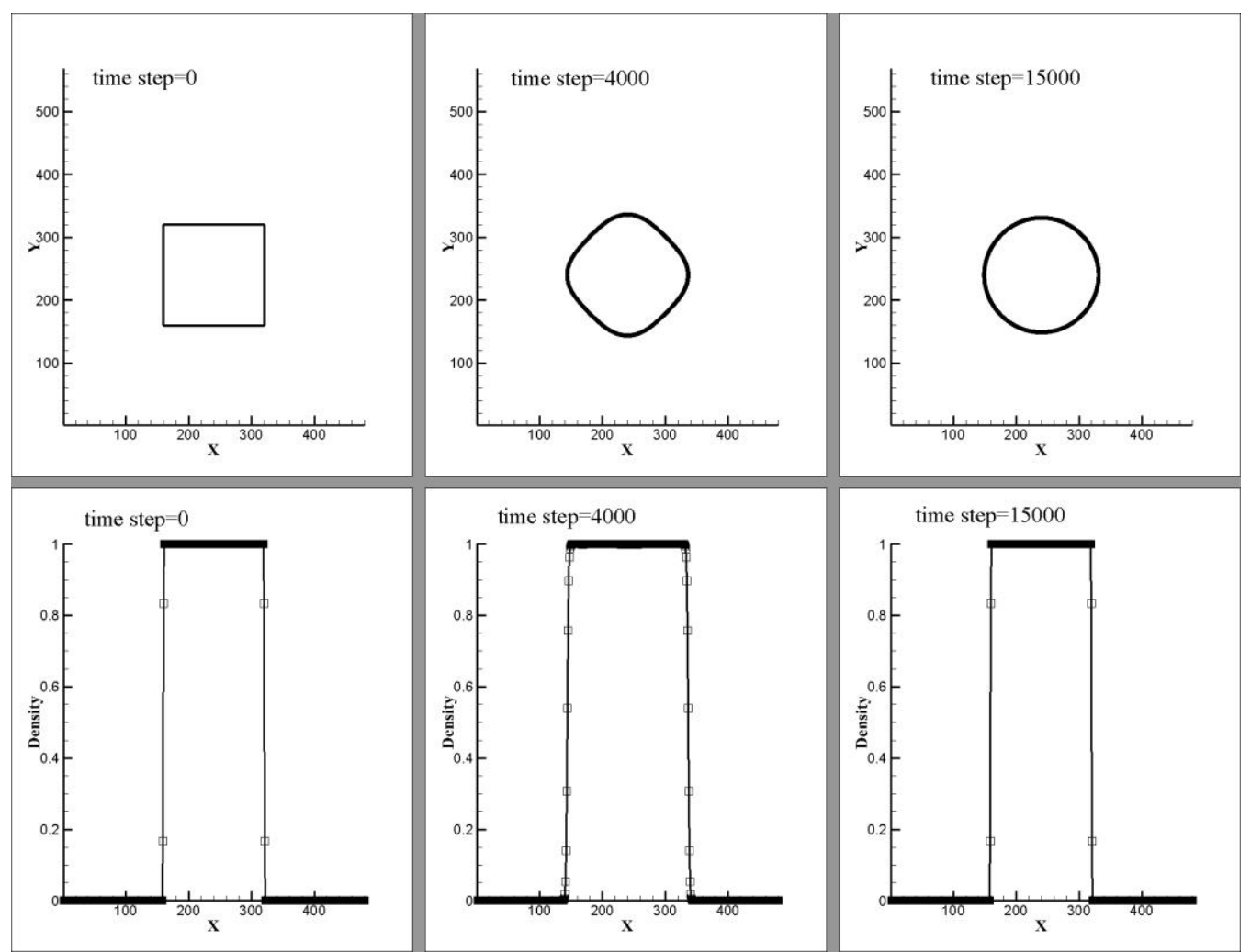

Fig. 5. Square drop relaxation test, drop shape evolution, and its density variation at the time steps $t=0$, 4000 , and 15000 for $480 * 480$ grid.

drop growth process. The geometrical non-linearity is one of the primary sources in the dynamical system non-linearity. During drop growth, the contact angle between the growing drop and 
the pipe tip begins to increase. Increasing contact angel causes an increase in vertical component of surface tension force, which cancels out the drop weight. The process of increasing vertical component of the surface tension force continues until the contact angel becomes right (maximum of the vertical component of surface tension force), which is the end of the hardening region. By advancing the contact angle to more than the right angle, the vertical component of surface tension force begins to decrease (beginning of softening region). The region in the vicinity of the inflection point of FDFPD may be called pseudo-linear region. So, the overall geometry of pendent drop evolves with time, which causes its non-linear behavior. Further illustrative explanations are given in Section A.2 of the appendix. In all of the simulations, it was realized that the local maximum point of FDFPD could be considered as a pinch off criterion.

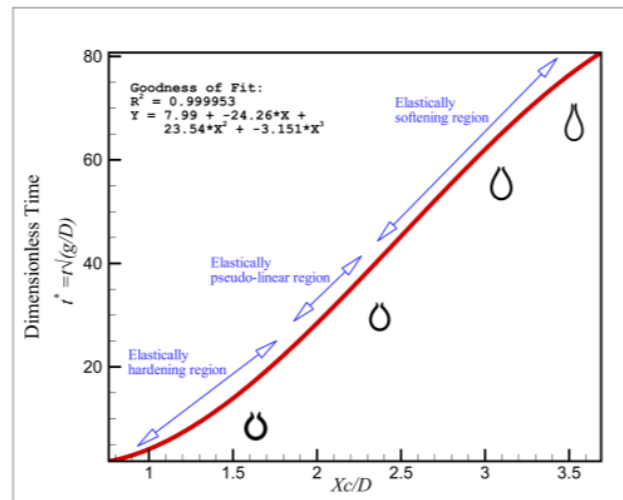

(a)

Dimensionless Drop Center Height

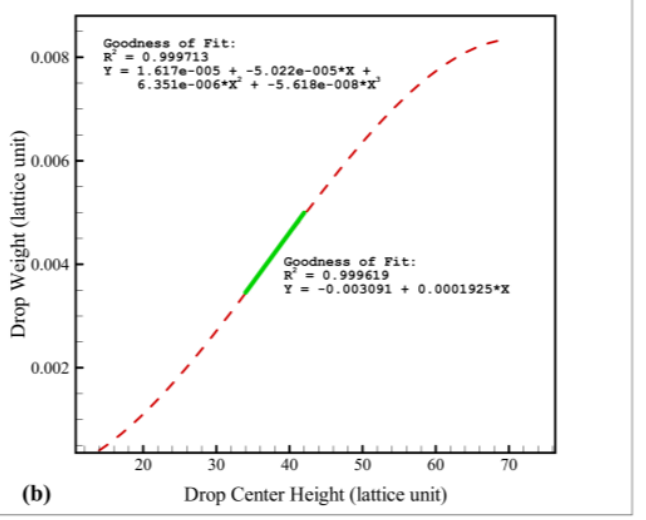

Fig. 6. Numerically derived Force- displacement function of pendent drop (FDFPD) for the growing drop during its formation and detachment (a) three different elastic regions of FDFPD (dimensionless axes), (b) regression line fitted to the linear region of FDFPD to calculate the linear elastic coefficient of the drop, $k=1.925 \mathrm{E}-4$, (axes in lattice units).

Extensive experiments on the formation and detachment of drops were performed by (Zhang and Basaran 1995). They illustrated the variations of drop neck thickness versus drop formation time for water in air. Their results are shown in Figs. 6 and 8 (Zhang and Basaran 1995). As it is shown in these figures, the trends for variations of surface tension force of growing pendent drop are the same as in Fig. 6. The simulation was performed with surface tension coefficient $\sigma=0.004$, inner pipe diameter $D=14$, density ratio of 1,000 , inlet velocity of $\mathrm{U}=$ $1.1 \mathrm{E}-5$, and gravitational acceleration of $\mathrm{g}=2.28 \mathrm{E}-$ 6. All the quantities are in the lattice units.

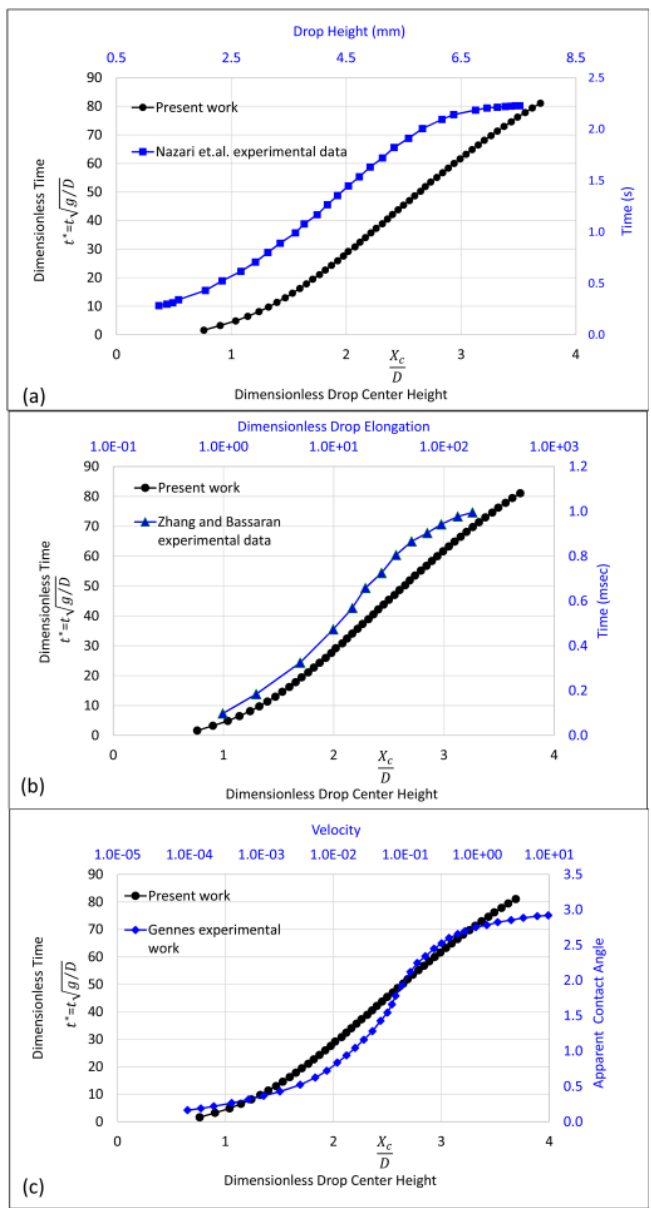

Fig. 7. Comparison of the trends between the Fig. 6 of this work with a) Fig. 5 of Nazari $e t a l$. (2018), b) Fig. 8 of Zhang and Bassaran (1995), c) Fig. 27 of Gennes (1985).

Jones concluded that the surface tension force has a non-linear nature (Jones, 1996). Jones exploiting figure 4.26 of (Jones, 1996), realized that at the beginning of drop formation, surface tension force increases, and after a certain point, it starts to decrease. This conclusion compares well with the result of this work about the variation of surface tension force, as illustrated in Fig. 6. Here it is appropriate to explain the relation of the graph to the surface tension force. The ordinate of the curve in Fig. 6 is the time step of simulation, which has been non-dimensionalized. During the process of the drop growth, two opposite forces are in balance, namely drop weight and surface tension force. So, 
there is a corresponding mass and, consequently, a weight force for each time step, which increases during the drop growth. Gennes studied the Mechanics of wetting phenomena experimentally (de Gennes 1985).

A precise issue referred to in that work is the experimental curve (after (Hoffman 1975)) relating apparent contact angle variations to the speed of drop interface motion. Gennes used silicon oil drop on glass and generated Fig. 27 of (de Gennes 1985). Therein, the variation of surface tension force is such that it consists of three distinct regions (hardening, pseudo-linear, and softening) during drop formation. As it is seen, there is a close coincidence between the trends of Fig. 6 and the curve presented by Gennes. One of the recent works has been performed by Nazari et al. (Nazari et al. 2018). In this experimental work, they have investigated the growing drop profiles using a highspeed camera and generated a curve that relates the drop mass center to the time during drop growth and formation. In Fig. 5, the trend is in full agreement with Fig. 6 of the present work, which describes force-displacement of a growing drop. Figure 7a,b, and c illustrates the comparison between Fig. 6 of this work and 5 of Nazari et al. for water in n-Hexane, 8 of Zhang and Bassaran, and 27 of Gennes, repectively. The difference between the curves is due to the fact that the one related to this work is dimensionless, while the one presented by Nazari et al. is not. If the forcedisplacement diagram of pendent drop for $\mathrm{Re}=0.023$ and $\mathrm{Bo}=0.48$ (illustrated in Fig. 11) is plotted along with the one presented by Nazari et al. (Fig. A2).

\section{RESULTS AND DISCISSION}

\subsection{Force-Displacement Function}

By entering the denser fluid into the gas region, the drop mass center changes its position along the flow direction. This situation is very similar to hardening a spring elongated with time by increasing a force exerted on it. We can then draw a forcedisplacement curve for its growth (Fig. 6). In our extensive numerical results, we found that the process of formation and detachment of drop for any density ratios obeys the same non-linear forcedisplacement equation referred by Eq. (25), i.e.,

$\left\{\begin{array}{l}f(y)=\alpha+\beta y+\gamma y^{2}+\varepsilon y^{3}, \\ \varepsilon<0,\end{array}\right.$

where $\alpha, \beta, \gamma$, and $\varepsilon$ are real constants.

Force-displacement function of a pendent drop (FDFPD) for simulation of Fig. 6a, is presented by the Eq. (26).

$$
\begin{gathered}
f(y)=7.99-24.26 y \\
+23.54 y^{2}-3.15 y^{3}
\end{gathered}
$$

\subsection{Resonance of a Pendent Drop}

In non-linear vibrations (Kelly 2012; Yang, Sanjuán, \& Liu, 2016), there exist superharmonic and subharmonic resonances. When the excitation frequency is one-third of the system's natural linear region frequency, there is a superharmonic frequency in a system with cubic non-linearity. Also, there is a subharmonic frequency that is approximately three times the excitation frequency of the linear natural frequency of the system (Kelly 1996, 2012). To get the frequencies, the natural frequency was extracted from the linear region in the vicinity of the inflection point of Fig. 6b. For the simulation illustrated in Fig. 6a, this linear region occurs at about $t^{*}=42$, where $t^{*}$ is dimensionless time. By linear interpolating of data between $t^{*}=37$ and $t^{*}=47$, the linear spring constant is $\bar{k}=1.925 \mathrm{E}-4$ (in lattice units according to Fig. $6 b)$ ). The averaged drop mass of simulation in that region is $\bar{m}=1791$ (in lattice units).

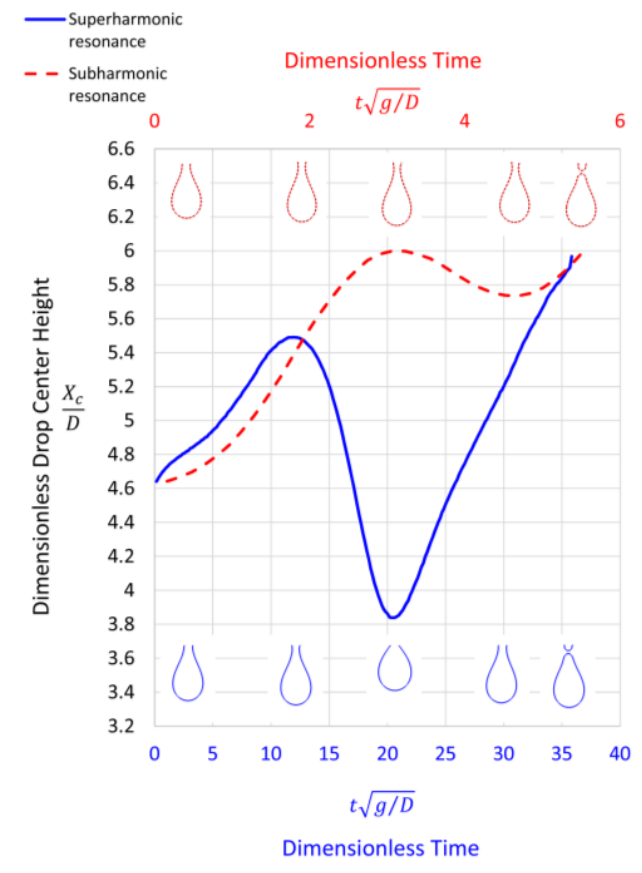

Fig. 8. Superharmonic and subharmonic resonances of simulated growing drop in Fig. 6a in its elastically softening region at $t^{*}=77.3$.

Then, simulation was considered using $\omega_{\text {sup }}=(\sqrt{ }(\bar{k} / \bar{m})) / 3$ and $\omega_{\text {sub }}=(3 \sqrt{ }(\bar{k} / \bar{m}))$ for two possible regions in the FDFPD. By selecting drop profile from softening region of Fig. 6 a at $t^{*}=77.3$ of the drop formation process, with surface tension $\sigma=0.004$, inner pipe diameter $D=14$, and density ratio of 1,000 , the drop was stimulated by an external sinusoidal force with superharmonic and subharmonic frequencies. It was observed that there is only one oscillation before drop detachment due to resonance in both frequencies (Fig. 8). Note, all the above quantities are in lattice units. The result is in agreement with the non-linear vibrations theory of superharmonic and sub-harmonic resonance. According to Fig. 8, in subharmonic resonance at about $t \sqrt{ }(g / D)=5.3$, the drop becomes unstable, and detaches. Also, in superharmonic resonance at about $t \sqrt{ }(g / D)=36$, the drop becomes unstable, and 
detachment occurs. Besides, in subharmonic resonance, the detachment time is about one-sixth of detachment time in superharmonic stimulation. The simulation was then repeated by a drop profile selected from the elastically hardening region at $t^{*}=40.5$ near the inflection point of FDFPD in Fig. 6.

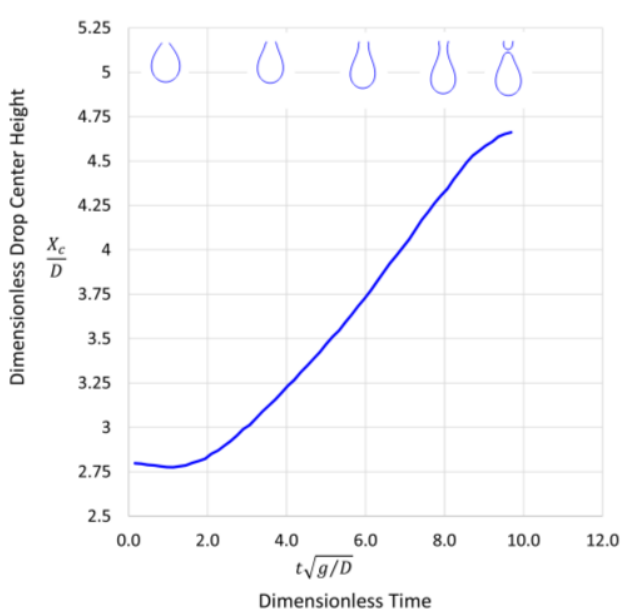

Fig. 9. Superharmonic resonance of the simulated growing drop in Fig. $6 a$ in its elastically hardening region at $t^{*}=40.5$.

In this region, It was realized that drop detachment was due to superharmonic resonance (subharmonic resonance was not observed). The external stimulation by changing drop geometry pushes it to enter softening region, which results in drop detachment. As a final remark, the close resemblance between Figs. 6 and 9 is interesting. Yet, they are representative of different physical phenomena. Figure 6 is due to a steady injection of mass under constant gravity force, and Fig. 9 (with no mass injection) is due to stimulation by an external sinusoidal force. So, we may say being in elastically softening region is a necessary condition for the drop detachment.

\subsection{Effect of Dimensionless Numbers}

We performed extensive simulations to examine the effect of the main dimensionless numbers engaged in drop formation, namely Reynolds, Capillary, and Bond numbers. Their definitions are presented in Section A.4 in the appendix. Note, capillary number is representative of relative importance of viscous force generated by internal flow to interfacial tension force. Since Reynolds number in these simulations are low, besides gravity the two forces, namely: surface tension and viscous, are the main effective and engaging forces in drop formation. As the heavier phase enters the lighter one, it is distorted by the viscous forces due to the relative velocity of the two phases. In fact, in many flow cases, in addition to the effect of gravity, these forces tend to extend and squeeze the drop downstream. (Gu et al. 2011). The surface tension force (which inherently tends to lower the interfacial area) tries to overcome viscous force by pulling the drop upstream. So, the overall shape of the pendent drop depends on the relative dominance of these forces. At low capillary numbers, surface tension force tends to dominate and the shape of the drop becomes spherical. In contrast, at high capillary numbers, viscous forces are dominant, resulting in deformation of the drop.

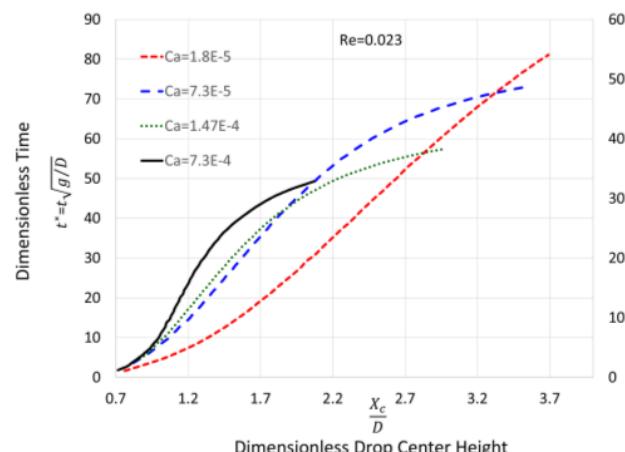

Fig. 10. Effect of Ca number variation with constant Reynolds number.

As a remark, the viscosity reduces drop oscillations at its interface due to its damping effects and stabilizes the drop. It almost has no effect on the drop size. According to Zhang and Basaran 1995 , the stabilizing effects of viscosity on drop formation have two aspects. First, viscosity damps the initial oscillations of the interface immediately remaining after the pinch-off of the previous drop at the tip of the tube. Second, viscosity has an essential effect in preserving the falling primary drop approximately in spherical shape as it is detaching from the pipe tip. For $\mathrm{Re}=0.023$ the Capillary number was set to $\mathrm{Ca}=1.8 \mathrm{E}-5, \mathrm{Ca}=7.3 \mathrm{E}$ $5, \mathrm{Ca}=1.47 \mathrm{E}-4$, and $\mathrm{Ca}=7.3 \mathrm{E}-4$. The results are shown in Fig. 10. As it is seen, increasing the $\mathrm{Ca}$ number (while keeping the Re number constant) results in the decrease of the detachment time (which is in good agreement with the results of (Zhang and Basaran 1995) and shrinking of linear portion in FDFPD. This phenomenon may be due to the weakening surface tension force effects in comparison to viscous effects that accelerate the detachment time. One possible explanation is that increasing the viscous effects increases the nonlinear character of the growing drop. As capillary number increases, viscous effects dominate and the drop stretches out. The more it extends, the more it deviates from its spherical shape (or symmetry), which causes increase in non-linearity due to geometrical changes. As explained earlier, the growing drop has a geometrical non-linearity.

Also, keeping the Reynolds number equal to 0.023 , other simulations were made by setting Bond number to $0.11,0.48,0.98$, and 1.96 . Interestingly enough, it was realized that by increasing Bond number, the linear portion of FDFPD enlarges and also the detachment time reduces (Fig. 11). This behavior may be due to the increase of gravitational forces effects that mainly have linear nature. Also, increasing Bond number means that relative importance of surface tension force decreases, causing detachment time to diminish. Obviously, keeping Reynolds number constant means we can 
not change dynamic viscosity, pipe diameter, and heavier phase velocity. So, the only way to change capillary number is changing surface tension coefficient. As a result, changing capillary number changed Bond number. Therefore, in Fig. 10 for capillary numbers of $1.8 \mathrm{E}-5,7.3 \mathrm{E}-5,1.47 \mathrm{E}-4$, and 7.3E-4, the corresponding Bond numbers are equal to $0.11,0.45,0.90$, and 4.5 , respectively, Also, changing Bond number was conducted by changing gravity. So, in Fig. 11 the capillary number was kept constant and equal to $1.8 \mathrm{E}-5$.

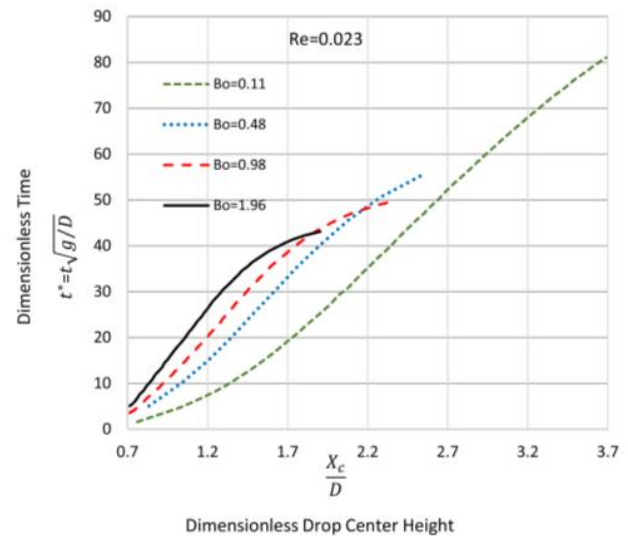

Fig. 11. Effect of Bond number variation at constant Reynolds number.

\section{CONCLUSION}

In this work, by introducing a new eqation, nonlinear elastic behavior of pendent drop was investigated. This equation fully expresses the dynamical specifications of the pendent drop (from growth to detachment). In addition, this equation makes it possible to identify elastic behavior of the pendent drop in regions having hardening, and softening elasticity by using its extrema and inflection points. The study of drop growth showed that the drop detachment occurs at the maximum point of this equation when the growing drop advances to the elastically softening region (by crossing the inflection point). In examining the oscillating behavior of the pendent drop, the superharmonic and subharmonic frequencies of the drop were determined using the natural frequency extracted from the linear region of the above mentioned equation. The pendent drop was resonated using superharmonic and subharmonic frequencies. It was observed that, first, the drop detachment occurs after its entrance to the elastically softening region. Second, when stimulating drop with subharmonic frequency, it detaches faster than when resonated with superharmonic one. The effect of capillary and Bond numbers on non-linear behavior of pendent drop detachment were also investigated. In this regard, it was observed that increasing capillary number reduces pinch-off time and increases nonlinear behavior of the drop, where the increase of Band number decreases both separation time and non-linear behavior of the drop.

\section{APPENDIX}

\section{A1. Lattice Parameters}

In this study, D2Q9 lattice was used is as follows (Fakhari and Bolster 2017):

$$
\left.\begin{array}{l}
\mathbf{e}_{0}=c(0,0) \\
\mathbf{e}_{1}=c(1,0) \\
\mathbf{e}_{2}=c(0,1) \\
\mathbf{e}_{3}=c(-1,0) \\
\mathbf{e}_{4}=c(0,-1)
\end{array}\right\} \quad w_{0}=4 / 9,
$$

where $c=\Delta x / \Delta t$, for the uniform grid $(\Delta x=\Delta t=1)$, in which $\Delta x$ and $\Delta t$ are lattice length and time scales, respectively, and $w$ 's are weight coefficients derived using Gauss-Hermit quadrature rule.

Using $\hat{\mathbf{S}}$ and $\mathbf{M}$ for D2Q9 from (Fakhari et al. 2017) $\mathbf{M}^{-1} \hat{\mathbf{S}} \mathbf{M}$ for 2D lattice is as follows:

$$
\mathbf{M}^{-1} \hat{\mathbf{S}} \mathbf{M}=\left[\begin{array}{cc}
\mathbf{T 1} & \mathbf{0} \\
\mathbf{0} & \mathbf{T 2}
\end{array}\right]
$$

where

$$
\mathbf{T 1}=\left[\begin{array}{ccccc}
1 & 0 & 0 & 0 & 0 \\
0 & \frac{s_{v}}{4}+\frac{3}{4} & \frac{1}{4}-\frac{s_{v}}{4} & \frac{1}{4}-\frac{s_{v}}{4} & \frac{1}{4}-\frac{s_{v}}{4} \\
0 & \frac{1}{4}-\frac{s_{v}}{4} & \frac{s_{v}}{4}+\frac{3}{4} & \frac{1}{4}-\frac{s_{v}}{4} & \frac{1}{4}-\frac{s_{v}}{4} \\
0 & \frac{1}{4}-\frac{s_{v}}{4} & \frac{1}{4}-\frac{s_{v}}{4} & \frac{s_{v}}{4}+\frac{3}{4} & \frac{1}{4}-\frac{s_{v}}{4} \\
0 & \frac{1}{4}-\frac{s_{v}}{4} & \frac{1}{4}-\frac{s_{v}}{4} & \frac{1}{4}-\frac{s_{v}}{4} & \frac{s_{v}}{4}+\frac{3}{4}
\end{array}\right],
$$

and

$\mathbf{T} 2=\left[\begin{array}{llll}\frac{s_{v}}{4}+\frac{3}{4} & \frac{1}{4}-\frac{s_{v}}{4} & \frac{1}{4}-\frac{s_{v}}{4} & \frac{1}{4}-\frac{s_{v}}{4} \\ \frac{1}{4}-\frac{s_{v}}{4} & \frac{s_{v}}{4}+\frac{3}{4} & \frac{1}{4}-\frac{s_{v}}{4} & \frac{1}{4}-\frac{s_{v}}{4} \\ \frac{1}{4}-\frac{s_{v}}{4} & \frac{1}{4}-\frac{s_{v}}{4} & \frac{s_{v}}{4}+\frac{3}{4} & \frac{1}{4}-\frac{s_{v}}{4} \\ \frac{1}{4}-\frac{s_{v}}{4} & \frac{1}{4}-\frac{s_{v}}{4} & \frac{1}{4}-\frac{s_{v}}{4} & \frac{s_{v}}{4}+\frac{3}{4}\end{array}\right]$.

In Eqs. (A3-A4), is defined as:

$$
s_{v}=\frac{1}{\tau+1 / 2}
$$

Where $\tau$ is the hydrodynamic relaxation time and is related to kinematic viscosity as: 
$v=\tau c_{s}^{2} \Delta t$

$\tau$ can be calculated using the following interpolation relation:

$\tau=\tau_{L}+\varphi\left(\tau_{H}-\tau_{L}\right)$

\section{A.2 Variation of Surface Tension Force with Appearant Contact Angle.}

Figure A.1 shows a pendent drop from a capillary tip. The growing drop has an apparent angle of $\theta$ with respect to the horizontal level of the capillary tip.

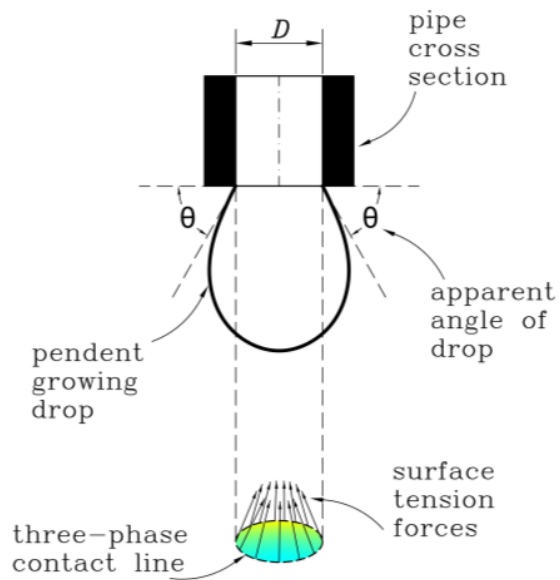

Fig. A1. Schematic of apparent angle of a growing drop. The drop has an angle of $\theta$ with a capillary tip. Here, the direction of surface tension forces acting on the three-phase contact line is shown.

Figure A1 shows surface tension force, $F_{s}$, which has an angle $\theta$ with the horizontal direction. This force exerts to the perimeter of the capillary tip with a diameter $D$, which is called the three-phase contact line. Its overall magnitude is $\pi \sigma D$, so its net magnitude in the vertical direction is $\pi \sigma D \operatorname{Sin} \theta$. In this regard, the variation of surface tension force can be considered as a function of $\theta$. While, the drop is growing, if $0<\theta<\pi / 2$ then surface tension force will be ascending, and if $\pi / 2<\theta<\pi$, it will be descending

\section{A.3 FDFPD with $\mathrm{Re}=0.023$ and $\mathrm{Bo}=0.48$}

In this section the FDFPD is illustrated for $\mathrm{Re}=0.023$ and $\mathrm{Bo}=0.48$ and compared with the Fig. 5 of Nazari et al.

\section{A.4 Dimensionless Numbers}

According to (Cramer 2004), there are mainly five important dimensionless numbers engaged in the drop formation problem, namely: Capillary $(\mathrm{Ca})$, Ohnesorge ( $\mathrm{Oh})$, Reynolds $(\mathrm{Re})$, Bond $(\mathrm{Bo})$, and viscosity ratio. Though $O h=\sqrt{ }(\mathrm{Ca} / \mathrm{Re})$, the three dimensionless numbers, $R e, B o$, and $C a$, were considered in this simulation. Their definitions are as:

$$
\begin{aligned}
& \operatorname{Re}=\frac{U D}{v}=\frac{\text { Inertial Forces }}{\text { Viscous Forces }}, \\
& C a=\frac{\rho v U}{\sigma}=\frac{\text { Fiscous Forces }}{\text { Surface Tension Forces }},
\end{aligned}
$$

Bo $=\frac{g \Delta \rho D^{2}}{\sigma}=\frac{\text { Gravitational Forces }}{\text { Surface Tension Forces }}$

where $U$ is heavier phase velocity entering from the pipe, $D$ is pipe inner diameter, $v$ is kinematic viscosity, $\rho$ is heavier phase density, $\Delta \rho$ is the twophase density difference, $g$ is gravity acceleration, and $\sigma$ is the surface tension coefficient.

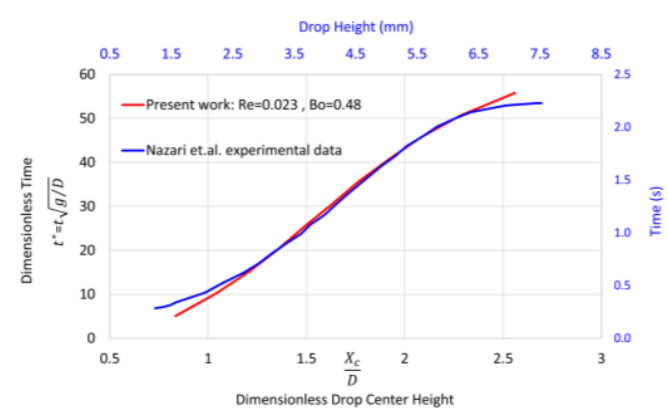

Fig. A2. Comparison of the trends between the FDFPD for $\mathrm{Re}=0.023$ and $\mathrm{Bo}=0.48$ with Fig. 5 of Nazari et al. (2018).

\section{ACKNOWLEDGEMENTS}

This research did not receive any specific grant from funding agencies in the public, commercial, or not-for-profit sectors.

\section{REFERENCES}

Allen, S. M. and J. W. Cahn(1979). A microscopic theory for antiphase boundary motion and its application to antiphase domain coarsening. Acta Metallurgica 27(6), 1085-1095.

Bierbrauer, F., N. Kapur and M. C. T. Wilson (2013). Drop Pinch-Off for Discrete Flows from a Capillary. ESAIM: Proceedings 40(February), 16-33.

Bostwick, J. B. and P. H. Steen (2009). Capillary oscillations of a constrained liquid drop. Physics of Fluids 21(3), 032108.

Cahn, J. E. and J. W. Hilliard (1958). Free Energy of a Nonuniform System. II. Interfacial Free EnergyThermodynamic Basis. The Journal of Chemical Physics 28(2), 258-267.

Chen, S., D. Martínez and R. Mei (1996). On boundary conditions in lattice Boltzmann methods. Physics of Fluids 8(9), 2527-2536.

Chiu, P. H. and Y. T. Lin (2011). A conservative phase field method for solving incompressible two-phase flows. Journal of Computational 
Physics 230(1), 185-204.

Clift, R., J. R. Grace and M. E. Weber (1978). Bubbles, Drops, and Particles. New York: Academic Press.

Cramer, C. (2004). Continuous Drop Formation at a Capillary Tip and Drop deformation in a Flow Channel. Zurich: Ph.D Dissertation.

d'Innocenzo, A. and L. Renna (1996). Analytical solution of the dripping faucet dynamics. Physics Letters A 220(1-3), 75-80.

Davidson, M. and J. Copper-White (2003). Numerical prediction of shear-thinning drop formation. 3rd International Conference on CFD in the Minerals and Process Industries (December), 403-408.

de Gennes, P. G. (1985). Wetting: statics and dynamics. Reviews of Modern Physics 57(3), 827-863.

DePaoli, D. W., J. Q. Feng, O. A. Basaran and T. C. Scott (1995). Hysteresis in forced oscillations of pendant drops. Physics of Fluids 7(6) 11811183.

Fainerman, V. B. and R. Miller (1995) Hydrodynamic effects in measurements with the drop volume technique at small drop times. 2. Drop time and drop volume bifurcations. Colloids and Surfaces A: Physicochemical and Engineering Aspects 97(3), 255-262.

Fakhari, A. and D. Bolster (2017). Diffuse interface modeling of three-phase contact line dynamics on curved boundaries: A lattice Boltzmann model for large density and viscosity ratios. Journal of Computational Physics 334(November), 620-638.

Fakhari, A. and M. H. Rahimian (2009). Simulation of falling droplet by the lattice Boltzmann method. Communications in Nonlinear Science and Numerical Simulation 14(7), 3046-3055.

Fakhari, A. and M. H. Rahimian (2010). Phase-field modeling by the method of lattice boltzmann equations. Physical Review E - Statistical, Nonlinear, and Soft Matter Physics 81(3), 036707.

Fakhari, A. and M. H. Rahimian (2011). Investigation of deformation and breakup of a falling droplet using a multiple-relaxationtime lattice Boltzmann method. Computers and Fluids 40(1), 156-171.

Fakhari, A. and T. Lee (2013). Multiple-relaxationtime lattice Boltzmann method for immiscible fluids at high Reynolds numbers. Physical Review E - Statistical, Nonlinear, and Soft Matter Physics 87(2), 023304.

Fakhari, A., M. Geier and T. Lee (2016). A massconserving lattice Boltzmann method with dynamic grid refinement for immiscible twophase flows. Journal of Computational
Physics 315(April), 434-457.

Fakhari, A., T. Mitchell, C. Leonardi and D. Bolster(2017). Improved locality of the phasefield lattice-Boltzmann model for immiscible fluids at high density ratios. Physical Review E 96(5).

Fawehinmi, O. B., P. H. Gaskell, P. K. Jimack, N. Kapur and H. M. Thompson (2005). A combined experimental and computational fluid dynamics analysis of the dynamics of drop formation. Proceedings of the Institution of Mechanical Engineers, Part C: Journal of Mechanical Engineering Science 219(9), 933947.

Geier, M., A. Fakhari and T. Lee(2015). Conservative phase-field lattice Boltzmann model for interface tracking equation. Physical Review E - Statistical, Nonlinear, and Soft Matter Physics 91(6).

Gu, H., M. H. G. Duits and F. Mugele (2011). Droplets Formation and Merging in TwoPhase Flow Microfluidics. International Journal of Molecular Sciences 12(4), 25722597.

Hoffman, R. L. (1975). A study of the advancing interface. I. Interface shape in liquid-gas systems. Journal of Colloid and Interface Science 50(2), 228-241.

Jacqmin, D. (1999). Calculation of Two-Phase Navier-Stokes Flows Using Phase-Field Modeling. Journal of Computational Physics 155(1), 96-127.

Jacqmin, D. (2000). Contact-Line Dynamics of a Diffuse Fluid Interface. Journal of Fluid Mechanics 402, 57-88.

Jones, L. A., T. W. Eagar and J. H. Lang (1998). A dynamic model of drops detaching from a gas metal arc welding electrode. Journal of Physics D: Applied Physics 31(1), 107-123.

Jones, L. A. (1996). Dynamic electrode forces in gas metal arc welding. PhD Thesis, Massachusetts Institute of Technology.

Karbaschi, M., M. Taeibi Rahni, A. Javadi, C. L. Cronan, K. H. Schano, S. Faraji,... R. Miller (2015). Dynamics of Drops - Formation, Growth, Oscillation, Detachment, and Coalescence. Adv Colloid Interface 222, 413424.

Kelly, S. G. (1996). Schaum's outline of theory and problems of mechanical vibrations. McGrawHill. Retrieved from https://books. google.com/books?id=-HIUAAAAMAAJ

Kelly, S. G. (2012). Mechanical Vibrations: Theory and Applications. Stamford: Global Engineering.

Lau, B. and F. Mashayek (2001). Dynamics of Oscillating Drops with Thermocapillary Effects. Theoretical and Computational Fluid Dynamics 14(4), 203-222. 
S. Ghorbanifar et al. / JAFM, Vol. 14, No. 1, pp. 331-344, 2021.

Lee, T. (2009). Effects of incompressibility on the elimination of parasitic currents in the lattice Boltzmann equation method for binary fluids. Computers \& Mathematics with Applications 58(5), 987-994.

Miller, R., M. Bree and V. B. Fainerman (1998). Hydrodynamic effects in measurements with the drop volume technique at small drop times-3. Surface tensions of viscous liquids. Colloids and Surfaces A: Physicochemical and Engineering Aspects 142(2-3), 237-242.

Miller, R. and L. Liggieri (2011). Bubble and Drop Interfaces. In Section I (1st ed., p. 665). CRC Press.

Mitchell, T., C. Leonardi and A. Fakhari (2018). Development of a three-dimensional phasefield lattice Boltzmann method for the study of immiscible fluids at high density ratios. International Journal of Multiphase Flow 107, $1-15$.

Nazari, A., A. Zadkazemi Derakhshi, A. Nazari and B. Firoozabadi (2018). Drop formation from a capillary tube: Comparison of different bulk fluid on Newtonian drops and formation of Newtonian and non-Newtonian drops in air using image processing. International Journal of Heat and Mass Transfer 124, 912-919.

Shaw, R. (1984). The Dripping Faucet as a Model Chaotic System. Santa Cruz: Aerial Press.

Sultana, K. R., K. Pope, L. S. Lam and Y. S. Muzychka(2017). Phase change and droplet dynamics for a free falling water droplet. International Journal of Heat and Mass Transfer 115, 461-470.

Timgren, A., G. Trägårdh and C. Trägårdh (2009). Effects of cross-flow velocity, capillary pressure and oil viscosity on oil-in-water drop formation from a capillary. Chemical Engineering Science 64(6), 1111-1118.

Zhang, D. F. and H. A. Stone (1997). Drop formation in viscous flows at a vertical capillary tube. Physics of Fluids 9(8), 22342242.

Zhang, X. and O. A. Basaran (1995). An experimental study of dynamics of drop formation. Physics of Fluids 7(6), 1184-1203. 